\title{
Optimized Laser Thermal Pulsing of Atom Probe Tomography: LEAP 4000X ${ }^{\mathrm{TM}}$
}

\author{
J.H. Bunton, J.D. Olson, D.R. Lenz, D.J. Larson, and T.F. Kelly \\ Imago Scientific Instruments Corp., 5500 Nobel Drive, Madison, WI 53719-1193 USA
}

Extensive investigations into the mechanism of laser pulsing in atom probe tomography have shown that the dominant pulsing mechanism is thermal [1]. This was recently confirmed by independent study [2]. Optimization of thermal pulsing is therefore guided by the heat flow in the specimen. Heating times which are less than or equal to the electron thermalization time in materials ( $\sim 10 \mathrm{ps})$ can be achieved with lasers. In order to achieve the optimal performance with this mechanism, it is imperative that the smallest possible volume of material be heated so that rapid cooling ( $\sim 100 \mathrm{ps})$ may occur. Imago has used this design criterion to develop its second generation laser pulsing system: the LEAP 4000X.

Key new features of this system are: a beam conditioning unit, targeting assembly and in-vacuum optics to focus the laser beam to a smaller, diffraction-limited spot, a more precise automated beam alignment and focusing system to maintain the beam on the specimen apex during field evaporation, and a large dynamic range $\left(>10^{6}\right)$ of pulse energies, Fig 1 . The laser light is a third harmonic at 355 $\mathrm{nm}$ wavelength with a pulse duration of $10 \mathrm{ps}$ and adjustable pulse repetition rates up to $1 \mathrm{MHz}$. The diffraction-limited spot at the specimen has a $4 \sigma$ diameter less than 5 micron. This geometry makes it possible to achieve very high mass resolving power (FWHM>1900, FW.1M>950, FW.01M>525 at $\mathrm{m} / \mathrm{n}=27$ and $>100 \mathrm{~nm}$ field of view) on suitable specimens, Fig. 2.

A diffraction-limited spot and shorter wavelength mean smaller irradiated tip area. Shorter wavelength also leads to smaller heated depth because the anomalous skin depth decreases with wavelength in most materials $[3,4]$. The result is smaller heated volumes, faster cooling, and improved mass resolution. With the lower overall thermal load, $1 \mathrm{MHz}$ repetition rates are achieved with no rise in base temperature. This makes it possible to obtain 100 million atom datasets in $1 \mathrm{hr}$.

The benefits to the user are manifold. If there are significant variations in thermal absorption properties along the heated shank (layered structures, second phases, Pt-C bond from FIB), thermallag peaks that can appear in the mass spectrum are eliminated. A small heated volume minimizes migration of contamination along a shank (e.g., water, platinum from FIB joint) that produces contamination peaks and noise from these materials. By minimizing the overall thermal input to the specimen, a broad spectrum of materials including stainless steel, ceramic coatings, and multilayer structures and lift-out specimens may be analyzed with good performance. With the low noise, 1 appm detection of $\mathrm{B}$ and As has been demonstrated. The specimen yield has also been found to improve such that greater than $70 \%$ yield on lift-out specimens of single bit devices has been achieved. The combination of small focused spot and shorter wavelength produces the best possible data for all material types.

References

[1] J.H. Bunton et al., Microscopy and Microanalysis, Vol. 13, No. 6 (2007) pp. 418-427.

[2] F. Vurpillot et al., J. Phys. D: Appl. Phys. 42 (2009) 125502.

[3] G.L. Eesley, Phys. Rev. Lett. 51 (1983) 2140.

[4] H. Park, et al., Physical Review B 72 (2005) 100301(R). 


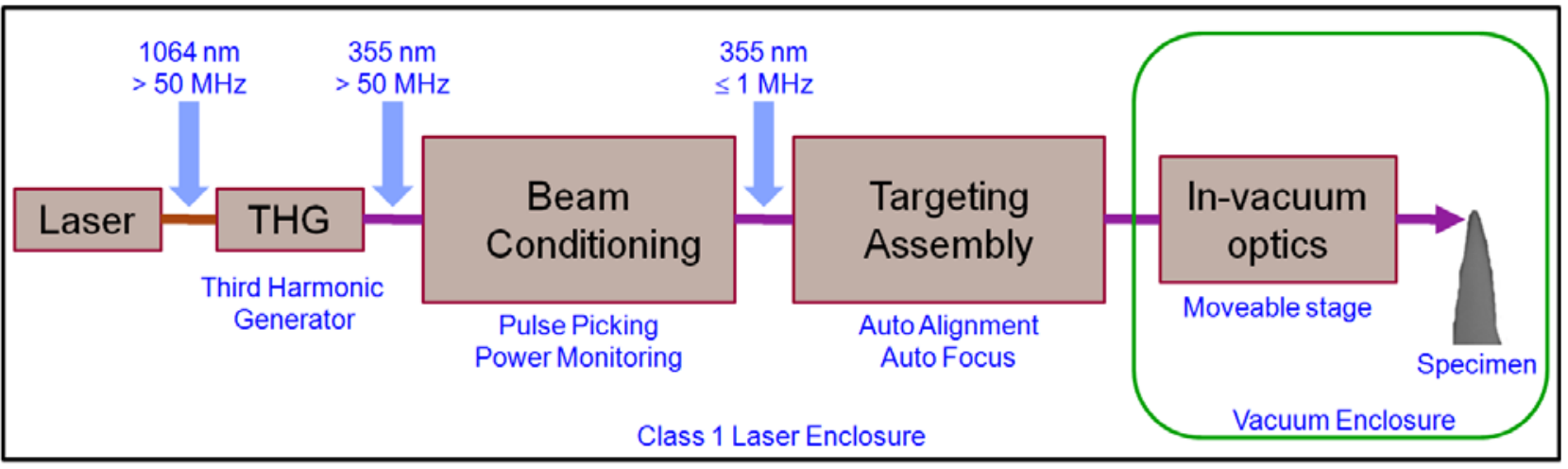

FIG. 1 Schematic of the LEAP 4000X laser pulsing system.

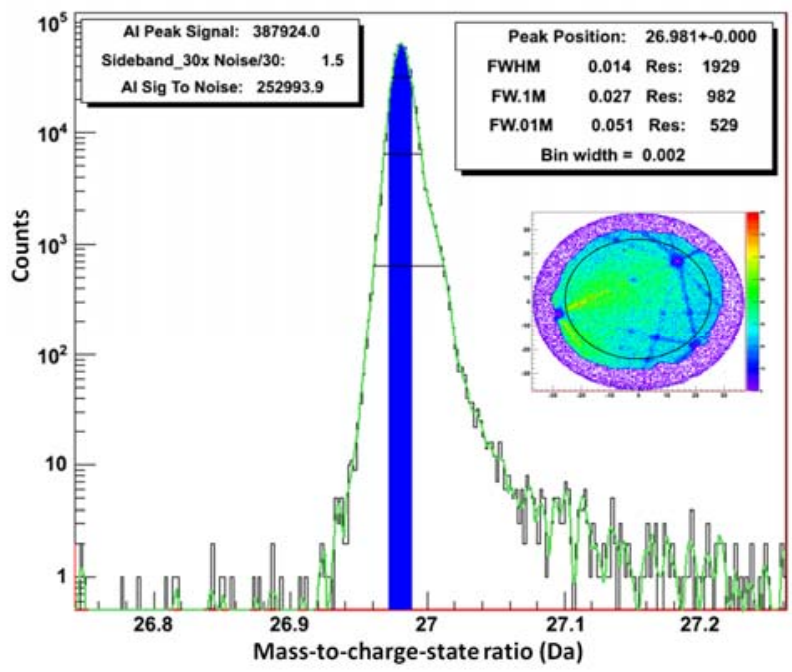

FIG. 2 Mass spectrum (vertical log scale) of aluminum showing high mass resolution achieved with the diffraction-limited spot in the LEAP at full field of view is better than 1/1900 FWHM.

a)

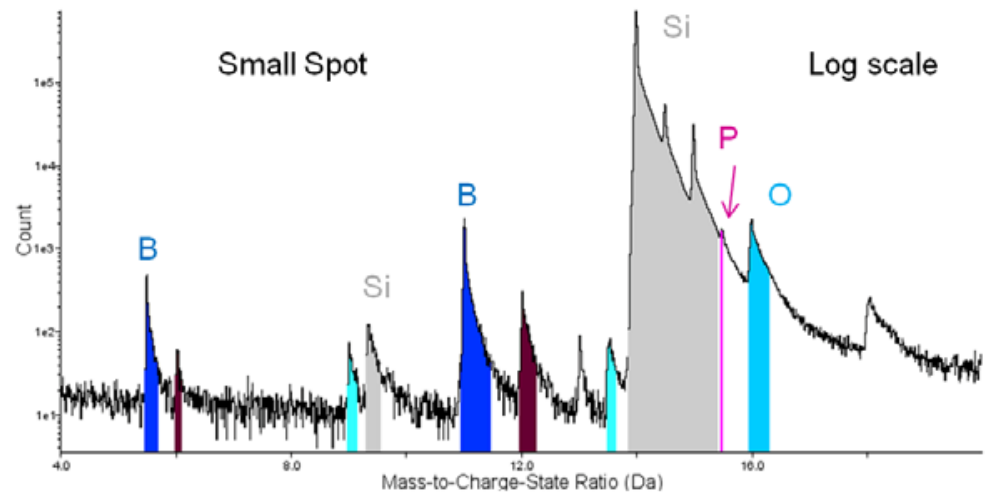

b)

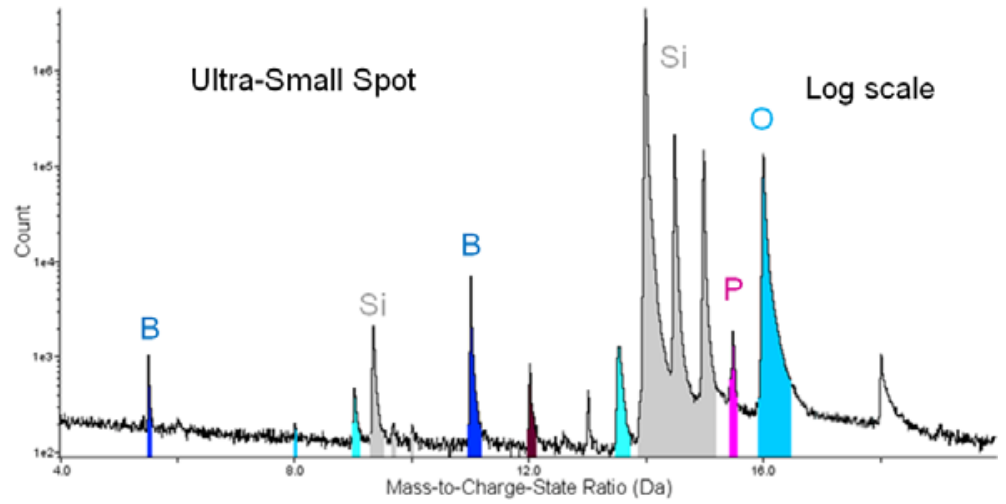

FIG. 3 Mass spectra (vertical log scale) showing the benefit of the focused spot for detection of low-concentration elements like phosphorus in silicon. Note the greatly reduced thermal tails in b). 